\title{
Dynamic Evaluation of Pelvic Floor Reconstructive Surgery Using Radiopaque Meshes and Three-dimensional Helical CT
}

\author{
Paulo Palma, Cassio Riccetto, Rogerio Fraga, Ricardo Miyaoka, Adilson Prando \\ Division of Urology (PP, CR, RF, RM), Universidade Estadual de Campinas, Unicamp, and Division \\ of Radiology (AP), Hospital Vera Cruz, Campinas, Sao Paulo, Brazil
}

\begin{abstract}
Purpose: This prospective study was performed to achieve visualization of the reestablishment of anatomy after reconstructive surgery in the different pelvic compartments with non-absorbable radiopaque meshes, providing valuable anatomic information for surgeons implanting meshes.

Materials and Methods: A total of 30 female patients with stress urinary incontinence (SUI), anterior and posterior vaginal wall prolapse, or both underwent surgical repair using radiopaque meshes after written informed consent. Patients with SUI underwent five different surgeries. Patients with anterior vaginal prolapse underwent a procedure using a combined pre-pubic and transobturator mesh, and those with posterior vaginal prolapse underwent posterior slingplasty. Three-dimensional reconstruction using helical CT was performed four weeks postoperatively.

Results: In all cases, the mesh was clearly visualized. Transobturator slings were shown at the midurethra, and the anchoring tails perforated the obturator foramen at the safety region. Mini-slings were in the proper place, and computed angiography revealed that the anchoring system was away from the obturator vessels. In patients undergoing procedure for anterior vaginal prolapse, both pre-pubic armpit and obturator slings were clearly seen and the mesh was in the proper position, supporting the bladder base and occluding the distal part of the urogenital hiatus. Transcoccygeal sacropexy revealed indirectly a well-supported "neo rectovaginal fascia" and the anchoring tails at the level of ischial spines.

Conclusion: Three-dimensional helical tomography images of the female pelvis using radiopaque meshes have a potential role in improving our understanding of pelvic floor reconstructive surgeries. These radiopaque meshes might be the basis of a new investigative methodology.
\end{abstract}

Key words: pelvic organ prolapse; prostheses and implants; surgical mesh; tomography, spiral computed Int Braz J Urol. 2010; 36: 209-17

\section{INTRODUCTION}

The understanding of physiopathological concepts of stress urinary incontinence (SUI) and pelvic organ prolapse (POP) has changed over the last few years; this has led to the development of new therapies and surgical techniques.

Minimally invasive synthetic slings became the preferred technique in the last decade and several factors have contributed to their rapid acceptance. There have been a number of conceptual changes in addition to the developments of biomaterials (1).

The integral theory according to Petros and Ulmsten emphasizes the need to "restore anatomy to restore the function" (2). The theory is based on the fact that organs are suspended by ligaments and pelvic muscles inserted into these ligaments. The muscles stretch the organs against the ligaments to give them 
shape and support. By a sequence of coordinated contraction and relaxation, the organs are closed (continence) or are opened out actively (emptying). Lax ligamentous insertion points therefore may cause not only prolapse, but also, symptoms of incontinence and abnormal emptying. The Integral System of diagnosis and surgery is based on a 3 zones classification (anterior, mid and posterior). The anterior zone is the one addressed when treating SUI and the goal is to restore the anatomy involving the external urethral ligament, which attaches the external meatus to the anterior surface of the pubic bone; the suburethral vagina (or Hammock); and the pubo-urethral ligament (2).

However, the blind passage of long needles and the pull-through of the sling have remained the key stages of all sling techniques.

Sling use has also brought about a series of new complications related to the tape and to the techniques, some of which have even been proven to be potentially lethal $(3,4)$. Most of the major complications are related to the blind nature of the needles passage (5).

On the other hand, recurrence of POP, especially at the anterior compartment, has led to the development of new meshes and kits for POP repair.

Live anatomy restoration, however, is very difficult to assess due to the limitation of available imaging procedures and because the type-I meshes are not radiopaque.

When surgery is performed, the anatomical results are estimated using prolapses grading systems that are often subjective (6). Ultrasound has been used to assess the results of these procedures, but it is usually operator-dependent and may produce low quality images. Therefore, a better tool would be helical computerized tomography using three-dimensional reconstruction techniques, because it provides a high quality image and gives a dynamic evaluation when performed during rest and straining. A 3D method of visualizing the pelvic floor could facilitate understanding of the anatomy and function of this complex part of the body (7).

This prospective study was carried out in order to visualize the anatomical reconstruction after stress urinary incontinence and prolapse surgery in different pelvic compartments with non-absorbable radiopaque meshes.

\section{MATERIALS AND METHODS}

Multi-slice computer tomography (MSCT) was used to obtain high-resolution computer tomography (CT) images of pelvic regions (dynamic pelvic CT) and multi-plane reformatted (MPR) images. Thirty patients underwent pelvic floor reconstructive surgery for stress urinary incontinence (SUI) and anterior or posterior vaginal wall prolapse using radiopaque meshes (Table-1). These special meshes were made with barium-impregnated borders.

Helical pelvic scanning was performed with 1 $\mathrm{mm}$ slice thickness during rest and during straining.

In patients with SUI and anterior vaginal prolapse (AVP), surgeries were performed with the aid of a cystography. Anterior meshes (NAZCA $\mathrm{TC}^{\circledR}$, Promedon, Argentina) were placed with positioning of posterior transobturator arms anchored to the arcus tendineus at each side and anterior arms were self anchored to the prepubic space. In patients who underwent posterior vaginal prolapse (PVP) repair, an enema and a vaginogram were performed to establish landmarks and facilitate comprehension of the anatomy. A posterior mesh (NAZCA R ${ }^{\circledR}$, Promedon, Argentina) was placed with arms fixed through the sacrospinous ligaments at each side. Puncture was carried out $3 \mathrm{~cm}$ lateral and bellow the anal orifice.

Using the volume data acquired, overlapped reconstruction was performed at a stand-alone workstation (General Electric Medical System, GE Healthcare) to generate the images.

All patients were evaluated four to six weeks after the surgical procedure.

\section{RESULTS}

We evaluated a total of 30 patients who underwent pelvic floor reconstructive surgeries.

The meshes were visualized accurately in all cases. Patients with SUI underwent Transobturator tape (TOT) or mini-sling (OPHIRA ${ }^{\circledR}$ - PROM$\mathrm{EDON}^{\mathrm{TM}}$ ) procedures. Transobturator slings were attached at the midurethra and throughout the obturator foramen, allowing for normal urethral mobility during 
Table 1 - Demographic data from patients and procedures.

\begin{tabular}{lcc}
\hline Condition & Procedure & N \\
\hline \multirow{2}{*}{ SUI } & Transobturator tape & 5 \\
& Prepubic sling & 2 \\
& Micro-sling & 8 \\
Recurrent SUI & Retropubic sling & 1 \\
AVP & Crossover transobturator sling & 2 \\
PVP & Transobturator cystocele repair & 5 \\
& Infra-coccygeal sacropexy & 7 \\
Total & Transcoccygeal sacropexy & 30 \\
\hline
\end{tabular}

$A V P=$ anterior vaginal prolapse $P V P=$ posterior vaginal prolapse; $S U I=$ stress urinary incontinence.

straining (Figure-1). Micro slings were attached with anchoring columns near the obturator foramen without passing through. The angiographic study showed that the sling was positioned away from the major pelvic vessels (Figure-2).

Pre-operative study of patients with anterior vaginal wall prolapse showed the bladder below the inferior border of the pubic bone. The NAZCA-TC ${ }^{\circledR}$ (Promedon, Argentina) mesh in both the prepubic and transobturator approaches was clearly seen at the right position, supporting the bladder base and occluding the distal portion of urogenital hiatus. During stress maneuver, the bladder was maintained above the inferior border of pubic bone, indicating a successful treatment (Figure-3).

For placement of posterior meshes, transcoccygeal and NAZCA-R ${ }^{\circledR}$ (Promedon, Argentina) sacropexy was performed, showing De Lancey's

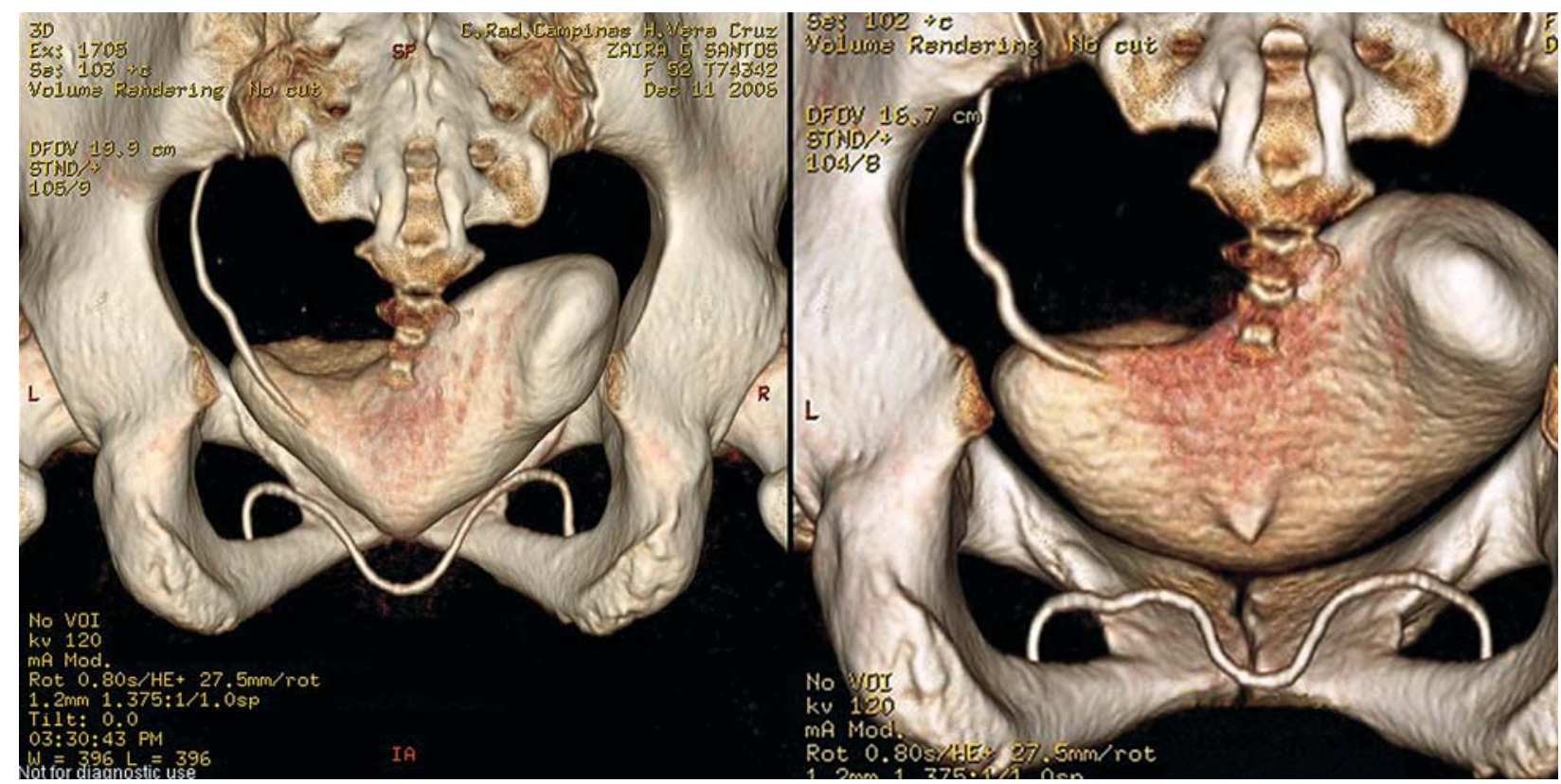

Figure 1 - Posterior views of a transobturator sling (Unitape ${ }^{\circledR}-$ Promedon $^{\mathrm{TM}}$ ) during strain and relaxation. 


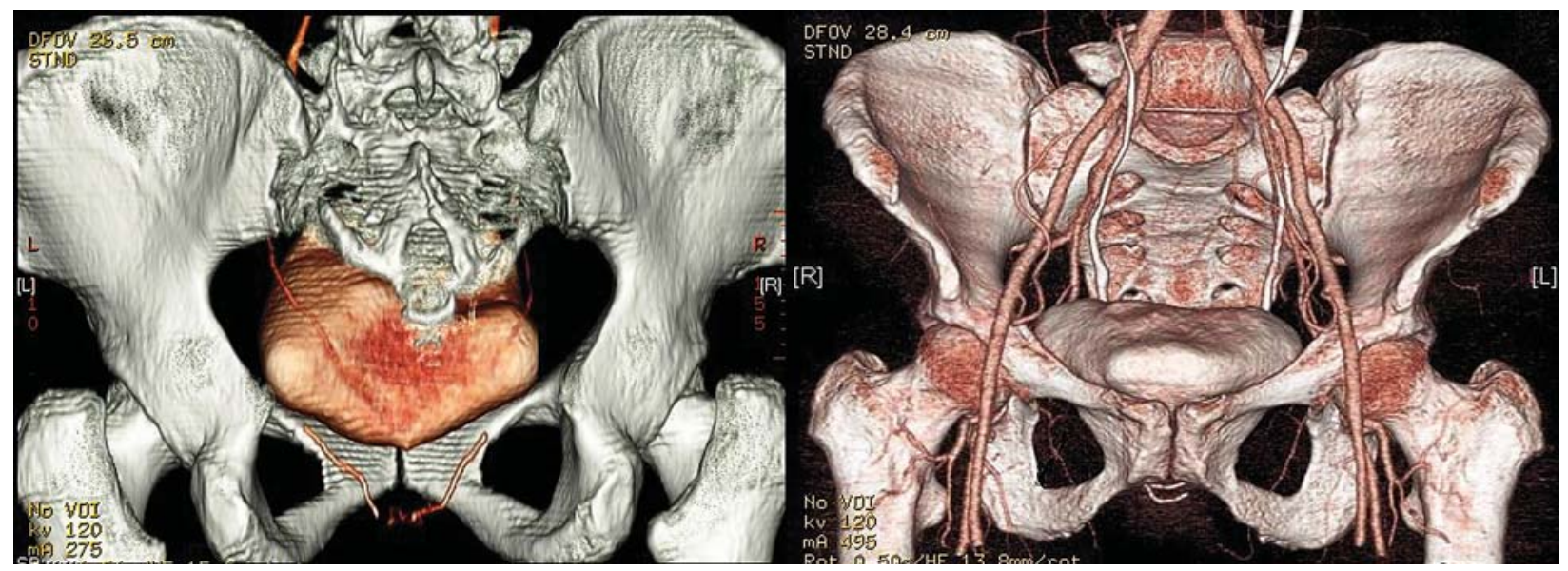

Figure 2 - Position of the Ophira ${ }^{\circledR}$ mini-sling and its distance from the obturator vessels.

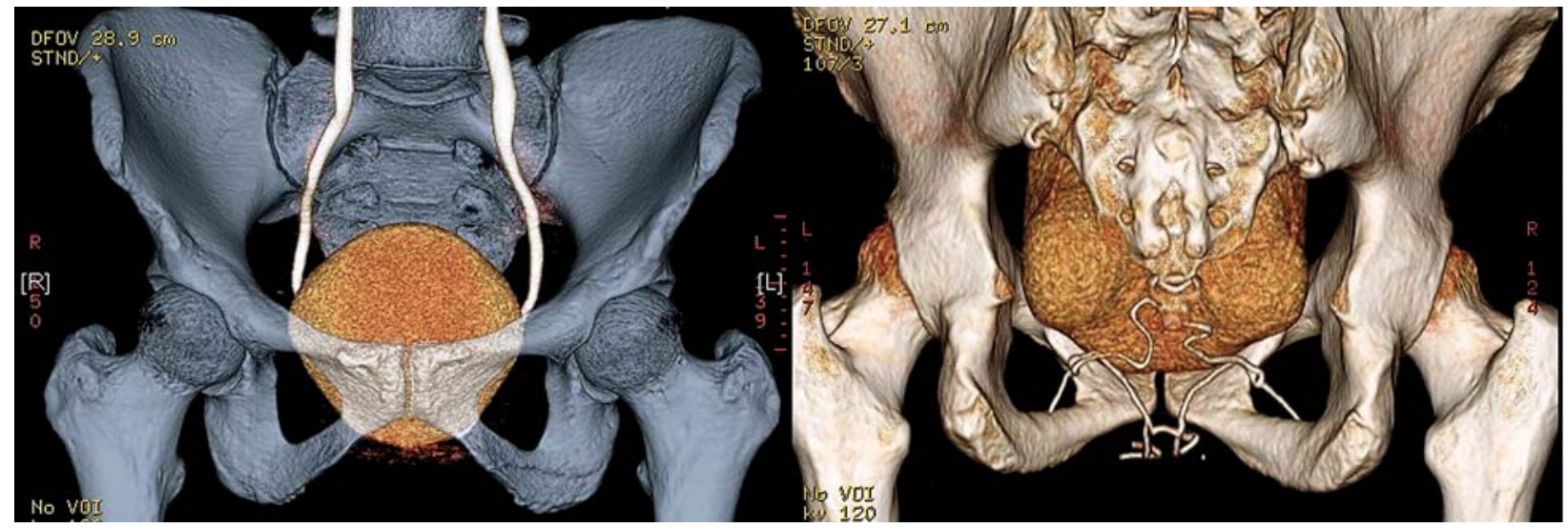

Figure 3 - Pre-and postoperative images of a cystocele corrected using Nazca-TC ${ }^{\circledR}$.

level I correction at the level of ischial spines (Figure-4).

There were no complications related to radiation exposure or contrast medium hypersensitivity.

\section{COMMENTS}

Female POP is the result of vaginal wall protrusion, associated or not with descensus uteri. The world's female population is becoming older as life expectancy becomes longer, and therefore the prevalence of POP is increasing (8). Thus, the risk for an 80 year-old woman to undergo treatment for POP or urinary incontinence is approximately $11 \%(9)$.

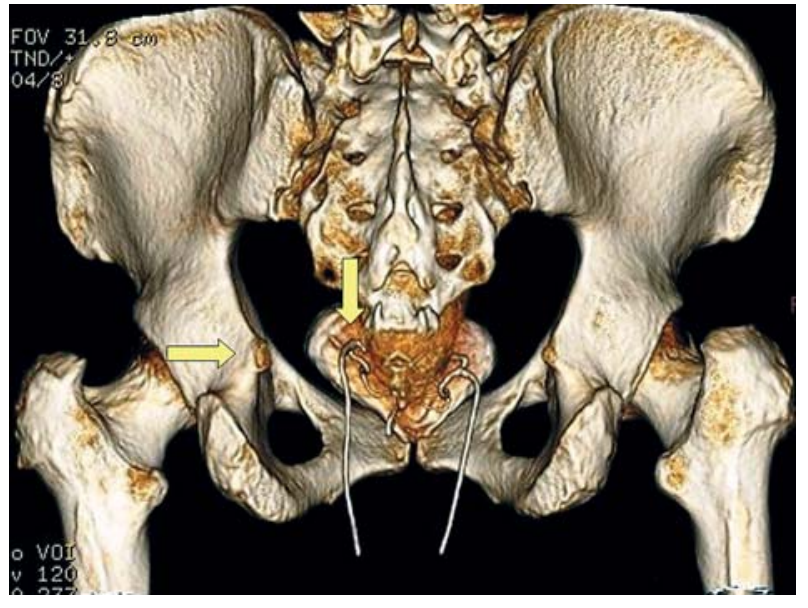

Figure 4 -Relationship between the Nazca- $R^{\circledR}$ and the ischial spine. 
The main techniques proposed to treat POP, levator ani myorrhaphy and pericervical ring repair, do not use synthetic materials such as polypropylene meshes. The high failure rate associated with these techniques could be explained by the poor tissue quality used in the surgical repair.

The use of synthetic meshes began in the last decade as an alternative to treat patients with high-grade prolapses and recurrent POP (10). Many attempts to demonstrate the live anatomy of these meshes have been made. Despite careful segmentation and verification by experts, limitations and some inaccuracies in ultrasound exist due to inherent technical limitations. The urethra and vagina, in particular, are small structures that are difficult to distinguish by transabdominal or transperineal ultrasound. Minor movement artifacts result in discrepancies in the quality of images (6).

In the present cases, dynamic pelvic $\mathrm{CT}$ and generation of MPR images allowed for direct observation of the pelvic floor adequate correction. Technologic advances in CT systems, including shorter scanning times with low radiation exposure and high quality images, allowed us to explore how the meshes work and how they behave under straining $(11,12)$.

Patients with SUI underwent two different types of surgeries for incontinence. Contrast medium and sometimes a Foley catheter were used to provide better visualization of the urethra. The TOT sling was clearly demonstrated at the medial border of the obturator foramen (safety zone), and a kinking in the slings was noticed during rest, confirming the low-tension procedure. During straining, the bladder neck moved downward to the inferior margin of the pubic bone, stretching the tape to create the backboard support with no leakage (Figure-1). The transobturator crossover sling was performed only as salvage procedure in patients with recurrent urinary incontinence due to severe intrinsic sphincter deficiency. In these cases, the slings assumed a helical shape around the midurethra, and the transobturator arms were observed (13). The detailed technique was described by Palma et al. and involves full dissection around the fixed urethra and placement of a "looping" sling anchored at each side through a transobturator arm (14). The Ophira mini-sling system ${ }^{\circledR}$, which supports the urethra in an arc-to-arc manner, was also displayed appropriately.
The tape was located at the midurethra, and the anchoring columns did not perforate the obturator foramen. The self anchoring short arms are placed through a minimal longitudinal incision underneath the mid urethra (15). During stress, normal mobility was preserved in accordance with the current concept of female stress incontinence.

A more detailed study using computed angiography showed the sling to be just below the pubis and the anchoring arms to be away from the obturator vessels (Figure-2). This confirms that the procedure may be performed in a safe manner, keeping the needle away from the neurovascular bundle.

Given the high recurrence rate of anterior vaginal prolapse with conventional repairs, synthetic meshes have been used in selected cases, such as high grade and recurrent prolapses (14-16).

CT scan was able to reveal pre-operatively a high-grade cystocele and post-operatively the body of the mesh supporting the bladder base and occluding the inferior part of the urogenital hiatus. During straining, the bladder neck remained above the inferior border of the pubic bone, confirming a good anatomical result (Figure-3).

Images obtained from the high-grade posterior vaginal wall prolapse correction showed the anchoring arm tips. The arrows point to the ischial spines, confirming the level I correction (Figure-4).

An enema was also performed in these patients, revealing a normal appearance of the rectum, which indicated proper correction of the rectocele.

\section{CONCLUSION}

The three dimensional helical tomography images of the female pelvis using radiopaque meshes have a potential role in improving our understanding of pelvic floor reconstructive surgeries. These radiopaque meshes might form the basis of a new investigative methodology.

\section{CONFLICT OF INTEREST}

Dr. Paulo Palma is a clinical investigator for Promedon, Argentina. 


\section{REFERENCES}

1. Norris JP, Breslin DS, Staskin DR: Use of synthetic material in sling surgery: a minimally invasive approach. J Endourol. 1996; 10: 227-30.

2. Petros PE, Ulmsten UI: An integral theory and its method for the diagnosis and management of female urinary incontinence. Scand J Urol Nephrol Suppl. 1993; 153: 1-93.

3. Bafghi A, Iannelli A, Trastour C, Bernard A, Ferroni A, Bongain A, et al.: Bowel perforation as late complication of tension-free vaginal tape. J Gynecol Obstet Biol Reprod (Paris). 2005; 34: 606-7.

4. Deng DY, Rutman M, Raz S, Rodriguez LV: Presentation and management of major complications of midurethral slings: Are complications under-reported? Neurourol Urodyn. 2007; 26: 46-52.

5. Petros PE, Richardson PA: Midurethral Tissue Fixation System sling -- a 'micromethod' for cure of stress incontinence -- preliminary report. Aust N Z J Obstet Gynaecol. 2005; 45: 372-5.

6. Parikh M, Rasmussen M, Brubaker L, Salomon C, Sakamoto K, Evenhouse R, et al.: Three dimensional virtual reality model of the normal female pelvic floor. Ann Biomed Eng. 2004; 32: 292-6.

7. Malmsten UG, Milsom I, Molander U, Norlén LJ: Urinary incontinence and lower urinary tract symptoms: an epidemiological study of men aged 45 to 99 years. J Urol. 1997; 158: 1733-7.

8. Gerten KA, Richter HE: Pelvic floor surgery in the older woman. Clin Obstet Gynecol. 2007; 50: 82643.

9. Olsen AL, Smith VJ, Bergstrom JO, Colling JC, Clark AL: Epidemiology of surgically managed pelvic organ prolapse and urinary incontinence. Obstet Gynecol. 1997; 89: 501-6.

10. de Tayrac R, Picone O, Chauveaud-Lambling A, Fernandez H: A 2-year anatomical and functional assessment of transvaginal rectocele repair using a polypropylene mesh. Int Urogynecol J Pelvic Floor Dysfunct. 2006; 17: 100-5.

11. Irwin DE, Milsom I, Hunskaar S, Reilly K, Kopp Z, Herschorn S, et al.: Population-based survey of urinary incontinence, overactive bladder, and other lower urinary tract symptoms in five countries: results of the EPIC study. Eur Urol. 2006; 50: 1306-14; discussion 1314-5.

12. Okamoto N, Maeda K, Kato R, Aoyama H, Hanai T, Sato $\mathrm{H}$, et al.: Enterocele associated with rectocele revealed by dynamic pelvic CT. Abdom Imaging. 2005; 30: 679-81.

13. Palma P, Riccetto CL, Dambros M, Fraga R, Maia R, Netto NR Jr: Evaluation of pelvic floor reconstructive surgery using tridimentional Helical CT. Eur Urol 2006; 5(Suppl.): 232. Abstract 910.

14. Palma P, Riccetto C, Herrmann V, Dambros M, Fraga R, Grossi O: Transobturator crossover readjustable sling for severe female incontinence: technique and preliminary results. Int Urogynecol J Pelvic Floor Dysfunct. 2007; 18: 1277-82.

15. Palma P, Riccetto C, Reges R, Fraga R, Miyaoka R, Hermann V, Marcondes T: Arcus to arcus microsling: technique and preliminary results. Int Urogynecol J Pelvic Floor Dysfunct. 2008; 19: 1133-6.

16. Palma P, Rane A, Riccetto C, Herrmann V, Dambros M, Thiel M, et al.: Correción transobturatória de los cistoceles. Actas Urol Esp. 2005; 29: 89-92.

\section{Correspondence address:}

Dr. Paulo Palma

Rua José Pugliesi Filho, 265

Campinas, SP, 13085-415, Brazil

E-mail: ppalma@uol.com.br 


\section{EDITORIAL COMMENT}

The authors report the use of helical CT to assess anatomical results following the surgical repair of stress urinary incontinence (SUI) and pelvic organ prolapse using a variety of mesh-based repairs. Accordingly, the authors radiographically confirm the expected post-operative site of these repair systems and demonstrate the correction of related pelvic floor defects. As described, previously used techniques have often provided images of limited quality and the authors succeed in providing high quality images following pelvic floor reconstruction. The added use of angiography and cystography, as well as the use of dynamic imaging during straining, adds to their visual findings.

Certainly, this study is of academic interest to those who specialize in the treatment of stress urinary incontinence and pelvic organ prolapse. Despite a significant body of research, I believe that many would characterize our understanding of the pathophysiology underlying stress urinary incontinence as limited. The authors describe two of the most notable anatomic theories of stress urinary incontinence. Indeed, such theories provide the framework for much of our current understanding of SUI and guide the development of novel techniques for surgical repair. Related to this work, I believe that the radiographic confirmation of a suburethral tape that stretches with strain so as to provide a suburethral support to a descending bladder neck is of great interest. Nonetheless, the exact relationship between anatomy and function is not clearly defined. Indeed, recent research identifies differing anatomic locations and relationships when comparing the anatomic support focal to the integral theory and that seen with midurethral slings (1). Such findings underscore the importance of future study to forward our understanding of urinary incontinence.

\section{REFERENCE}

1. Crivellaro S, Kocjancic E, Bussani R, Frea B: Are mid urethral slings the replacement of defective pubo urethral ligaments? An anatomic study. J Urol. 2009; 181(Suppl): 560 (abstract 1558).

\author{
Dr. David E. Rapp \\ Co-Director, Virginia Urology Center for \\ Continence and Pelvic Floor Prolapse \\ Seattle, Washington, USA \\ E-mail:derapp@yahoo.com
}

\section{EDITORIAL COMMENT}

From 1900 until the early 1990 s, surgery for stress incontinence (SI) and prolapse remained basically unchanged, either some type of vaginal or abdominal sling or suspension. Both types of operation were painful, and required catheters and hospital stays of 7 to 10 days. Complicating surgical management of SI was the convention of not operating on patients who had urodynamically demonstrated "detrusor instability", now known as "Overactive Bladder" (OAB) (1).
In 1990s the Integral Theory (2), and a method for creating artificial neoligaments were introduced (3). The theory stated that stress and urge symptoms in the female, both originated from lax suspensory ligaments of the vagina, and both were potentially curable surgically by creation of artificial ligaments. This method disregarded earlier urodynamically based conventions, and it has revolutionized the treatment of stress incontinence with minimal pain, short hospital 
and high long-term cure rates. Though apparently benign, major complications have been reported with these operations, provoking calls for zero tolerance for such operations (4).

The same surgical principle, creation of artificial neoligaments, has been applied to prolapse surgery. Beginning in 1992, polypropylene tapes inserted via the perineal route (infra-coccygeal sacropexy) were used to cure prolapse of the uterus (5). These methods did not address large cystoceles. Delorme's transobturator route (2001) for a midurethral sling was harnessed for repair of large cystoceles, and meshes were attached to tapes inserted via the perineal route for repair of large rectoceles.

Though effective, major problems have been encountered with the use of large meshes. Since 2005, new mini-sling techniques have been introduced to treat incontinence and prolapse (6-8). This method seems to avoid most of the problems of large mesh, using small lengths of tape to strengthen the natural tissues, rather than replace them totally.

This is a short summary of the rapid new direction in pelvic floor surgery, which has overtaken the Urogynecology community, all in the space of 15 years. Even the report of life-threatening complications, and attempts at caution (4) has not slowed the momentum of change.

Trailing a long way behind this accelerated innovation has been a precise understanding of where these meshes go, and their effect on pelvic floor structure and function. It is well and good to apply a theory, and to use it justify a particular incontinence or prolapse technique. But that is not good science. Good science is when a convention or theory is challenged forensically for truth or falsity in an objective way. That is the only way for medicine to progress.

Professor Palma and his team at Campinas University have been at the forefront of scientific challenge. Their editorial "The Dark Side of Urodynamics" (9) reinforced the Cochrane Review's conclusions, that pre-operative urodynamics have no predictive value for incontinence surgery. Invalidating the convention that urodynamically diagnosed "OAB" was a contraindication for surgery, has potentially opened the door for surgical cure for millions of women who have urgency symptoms. It has also indirectly



Figure 1 - A sitting $X$-ray taken in the straining position, with radio-opaque dye injected into the bladder $(B)$, vagina $(V)$, rectum (R), levator plate (LP). PUL and USL represent the pubourethral and uterosacral suspensory ligaments. The organs are tensioned like a trampoline by the muscle forces (arrows) contracting against the suspensory ligaments.

validated the Integral Theory's view that $\mathrm{OAB}$ is not a pathogenic condition per se. The Theory's view is that stress and urge both have anatomical origins, and urge and $\mathrm{OAB}$ are but different manifestations of a normal but prematurely activated micturition reflex caused by lax suspensory ligaments (10).

This forensic emphasis on functional anatomy is especially evident in the Palma team's teaching of diagnosis and surgery. They have widely taught the technique of "simulated" or "virtual" operations, a clinical challenge to the Theory (2), digitally anchoring specific ligaments during vaginal examination, and observing the effect. For example, pressing upwards to one side of the midurethra during coughing will control urine loss. Urge symptoms can also be reduced by pressing upwards in the position of other suspensory ligaments, uterosacral, and cardinal (gentle digital support of bladder base). They have constructed special teaching models for surgery, which mimic live pelvic floor anatomy (11).

Anatomical studies of tapes and implants in cadavers are not accurate, and are not validly transferable to the live patient. In the cadaver, all the muscles and organs are collapsed (12) while in the live patient (Figure-1), the organs are horizontal, suspended by 
ligaments and stretched by muscle forces like a trampoline (13).

The three-dimensional helical tomography technique using radiopaque meshes (14) is a major contribution to pelvic floor science, as it provides accurate reference points in the live patient, where the anatomy is similar to what is seen in Figure-1. For the first time, we have a precise tool to help us understand how the pelvic floor and its organs function, what implants do, and what they should not do. This is where the real worth of the new technique lies, its relevance to the live patient.

Though only the technique was presented in this contribution (14), it is certain that this method will be applied to answer so many questions left hanging by the whirlwind advance in surgical innovation we have witnessed over the past 15 years. With this contribution, Professor Palma and his team have demonstrated the necessity of good science as the essential monitor for innovation.

\section{REFERENCES}

1. Abrams P, Cardozo L, Fall M, Griffiths G, Rosier P, Ulmsten U, et al.: A The Standardization of Terminology of Lower Urinary Tract Function: Report from the Standardisation the Subcommittee- of the International Continence Society. Neurourol Urodyn. Neurourol Urodyn. 2002; 21: 167-78.

2. Petros PE, Ulmsten UI: An integral theory and its method for the diagnosis and management of female urinary incontinence. Scand J Urol Nephrol Suppl. 1993; 153: 1-93.

3. Petros PE, Ulmsten UI, Papadimitriou J: The autogenic ligament procedure: a technique for planned formation of an artificial neo-ligament. Acta Obstet Gynecol Scand Suppl. 1990; 153: 43-51.
4. Ostergard DR: The epochs and ethics of incontinence surgery: is the direction forward or backwards? Editorial. Int. Urogynecol J. 2002; 13: 1-3.

5. Petros PE: New ambulatory surgical methods using an anatomical classification of urinary dysfunction improve stress, urge and abnormal emptying. Int Urogynecol J Pelvic Floor Dysfunct. 1997; 8: 270-7.

6. Petros PE, Richardson PA: Midurethral tissue fixation system (TFS) sling for cure of stress incontinence--3 year results. Int Urogynecol J Pelvic Floor Dysfunct. 2008; 19: 869-71.

7. Petros PE, Richardson PA: The TFS mini-sling for uterine/vault prolapse repair: a three-year follow-up review. Aust N Z J Obstet Gynaecol. 2009; 49: 43940.

8. Petros PE, Richardson PA, Goeschen K, Abendstein B: The Tissue Fixation System provides a new structural method for cystocoele repair: a preliminary report. Aust N Z J Obstet Gynaecol. 2006; 46: 474-8.

9. Palma P, Herrmann V: Urodynamics and stress urinary incontinence: the dark side of a gold standard. Int Urogynecol J Pelvic Floor Dysfunct. 2007; 18: 1385-6.

10. Petros PE, Ulmsten U: Bladder instability in women: a premature activation of the micturition reflex. Neurourol Urodyn. 1993; 12: 235-9.

11. Palma P, Riccetto C, Fraga R, Portugal S, Dambros M, Rincón ME, Silveira A, Netto NR Jr: Three dimensional anatomy and virtual surgery for transobturator approach. Actas Urol Esp. 2007; 31: 361-5.

12. Zacharin RF: Pelvic Floor Anatomy and Cure of Pulsion Enterocoele. Wien, Springer-Verlag, 1985.

13. Petros PE, Ulmsten U: Role of the pelvic floor in bladder neck opening and closure: I muscle forces, II vagina. Int. J Urogynecol Pelvic Floor. 1997; 8: 69-80.

14. Palma P, Riccetto C, Fraga R, Miyaoka R, Prando A: Dynamic evaluation of pelvic floor reconstructive surgery using radiopaque meshes and tridimensional helical CT. Int Braz J Urol. 2009 (in press).

Dr. Peter E. Petros Department of Surgery Royal Perth Hospital Perth, Australia E-mail:kvinno@highwayl.com.au 